\title{
Metodologías inclusivas de aprendizaje mediante el uso de TIC: opiniones de estudiantes y docentes costarricenses
}

\section{Inclusive learning methodologies through use of ICT: Opinions of Costa Rican students and teachers}

\author{
Carolina Ávalos Dávila ${ }^{1}$ \\ Vivian Redondo Quesada \\ Daiyen Rojas Camacho \\ Jéssica Salazar Marín ${ }^{2}$ \\ Universidad Estatal a Distancia, Costa Rica
}

\begin{abstract}
Resumen. La presente investigación tiene como objetivo realizar un análisis comparativo de tres centros educativos, dos públicos y uno privado: el centro educativo Juan Flores Umaña, el CTP Fernando Volio y el centro educativo bilingüe Llama del Bosque respectivamente, con la finalidad de indagar sobre el uso de las diversas TIC usadas, en los procesos de enseñanza-aprendizaje en las áreas de ciencias e inglés, y valorar si tal uso apunta a la ejecución de metodologías de enseñanza y aprendizaje favorecedoras de una educación integral y de atención a la diversidad estudiantil en la población de sexto y de sétimo grado; para ello, se tomaron en cuenta sus similitudes, diferencias y formas de aprendizaje. La investigación de tipo mixto, exploratoria, descriptiva, fundamentada en el paradigma crítico y positivista, presenta los resultados, las opiniones de los docentes y estudiantes en cuanto al empleo de metodologías, técnicas y recursos TIC, en favor de generar espacios inclusivos de aprendizaje. Finalmente, se sensibiliza al cuerpo docente en el tema de inclusión educativa desde la capacitación de métodos que favorezcan procesos de aprendizaje integrales e inclusivos, como lo podría ser el método sobre Diseño Universal (DUA), favorecedor de metodologías de aprendizaje que, apoyadas por variados recursos tecnológicos, propicien una educación integral, innovadora y diversa.
\end{abstract}

Palabras clave. Inclusión educativa, proceso de enseñanza-aprendizaje, recursos TIC, metodologías y técnicas inclusivas

\begin{abstract}
The objective of this research is to carry out a comparative analysis of three educational centers, two public and one private: The Juan Flores Umaña School, the Fernando Volio CTP and the Llama del Bosque Bilingual High School respectively, with the purpose of investigating the use of the various ICTs used in the teaching-learning processes in areas of Science and English and assess whether such use, points to the implementation of teaching and learning methodologies favoring a comprehensive education and attention to student diversity in the population of sixth and seventh grade; taking into account their similarities, differences and ways of learning. Mixed, exploratory, descriptive research, based on the critical and positivist paradigm, presents from the results, the opinions of teachers and students regarding the use of methodologies, techniques and ICT resources used in favor of generating inclusive learning spaces. Finally, it is intended to sensitize the educators on the issue of educational inclusion from the training of methods that favor inclusive learning processes such as the Universal Design (DUA) method, as a support for learning methodologies that are supported by a variety of technological resources, promote an integral, innovative and diverse education.
\end{abstract}

Keywords. Educational inclusion, teaching-learning process, ICT resources, methodologies and inclusive techniques

${ }^{1}$ Carolina Ávalos Dávila. Máster en Currículum y Docencia Universitaria, y en Investigación e Innovación Educativa de la UNED España. Es candidata al Doctorado en Educación de la Universidad Nacional de Educación a Distancia en Madrid. Profesora de la Universidad Estatal a Distancia de Costa Rica, en la Escuela de Educación en las áreas de Didáctica, Currículo e Investigación Educativa. Correo electrónico: cavalos@uned.ac.cr

${ }^{2}$ Estudiantes graduadas del Bachillerato de Informática Educativa de la Universidad Estatal a Distancia de Costa Rica. Se desempeñan como docentes en el área de informática educativa en centros de educación primaria y secundaria. Correos electrónicos: viviredq@hotmail.com; dairojasc@gmail.com; jk-salazar@hotmail.com 


\section{Introducción}

Costa Rica es uno de los países de América Latina que, con el pasar de los años, se ha destacado por ofrecerles a sus estudiantes una educación con altos estándares de calidad. Aspecto que ha sido apoyado desde el Gobierno con la destinación de $8 \%$ de su producto interno bruto (PIB). Esto lo sitúa en el tercer país de Latinoamérica que más fondos destina al sector educación (Duryea y Robles, 2016).

Dicho aspecto ha permitido que se vayan disminuyendo brechas socioeconómicas, culturales y educativas, en las cuales la educación es cada vez una oportunidad de crecimiento para todos y, desde la Constitución Política, figura como la condición gratuita y obligatoria de mayor relevancia en el sector de educación primaria y secundaria, como lo sustenta el exministro de educación, Leonardo Garnier (2008):

Todos tienen derecho a una educación de calidad, que, partiendo de sus propias realidades, propicie el desarrollo de todo su potencial: estudiantes que aprenden a partir de estilos diferentes, con necesidades educativas especiales, talentosos, provenientes de distintos grupos étnicos, culturales, lingüísticos, que profesan credos religiosos diversos y cuyas condiciones sociales y económicas marcan sus ambientes de aprendizaje. (Ministerio de Educación, p.8).

Considerando lo anterior, el país tiene muy en cuenta la importancia de los espacios inclusivos de aprendizaje. Además, con una trayectoria superior a los 75 años en temas de educación especial y acuerpada con diferentes leyes en la misma temática, es capaz de brindar oportunidades a personas en condición de discapacidad, sensibilizando cada vez más a la sociedad actual en temas de diversidad y desarrollo. (Meléndez, 2015).

De acuerdo con aportes de Arnaiz (2003); Ainscow, Booth y Dyson (2006) y Ávalos (2015), los autores coinciden con que la educación inclusiva es una acción que involucra varios aspectos: sociales, culturales, políticos y educativos. Estos últimos deben converger para promover iniciativas que involucren la participación del estudiantado, así como otros agentes educativos que integran la comunidad de aprendizaje. De esta manera, se le da sentido a las siguientes interrogantes: ¿cómo se educa?, ¿qué espacios se consideran para el desarrollo de esa educación?, y ¿por qué se incurre en esa educación? Con ello se favorecen oportunidades de aprendizaje y de crecimiento donde la exclusión se vea disminuida.

En virtud de lo anterior, se resalta que la acción inclusiva debe estar presente como una práctica permanente en las aulas; sin embargo, su desarrollo implica una evolución necesaria en reformas de contenido, en metodologías de enseñanza y aprendizaje, en estrategias didácticas y en metodología de evaluación, cuya visión sea integrar los elementos curriculares e involucrar a toda la población estudiantil, como responsabilidad por parte del sistema educativo (UNESCO, 1994).

Investigaciones sobre inclusión educativa en Costa Rica han sido temas de mayor interés en los últimos años y se han convertido en una necesidad por atender en el campo educativo. Entre las indagaciones hechas, se destacan las de Marín y Meléndez (2011) sobre la transición de las instituciones educativas hacia la educación inclusiva, mediante el análisis de las actitudes, políticas y acciones institucionales en Costa Rica, quienes afirman que los proyectos desarrollados bajo una metodología inclusiva están en posibilidad de mejorar las condiciones educativas del estudiantado.

En otras, como la de Castro (2013), sobre implementación de prácticas inclusivas en instituciones educativas del Ministerio de Educación Pública en la región de Heredia, se 
analizaron las prácticas inclusivas desarrolladas en seis instituciones. Sus hallazgos han sido punto de inicio para que otras instituciones $\mathrm{y}$ regiones educativas reflexionen sobre el quehacer en este tema e inicien mejoras desde la práctica educativa.

Finalmente, en Segura y Martínez (2014), con el proyecto: Apoyo a la gestión pedagógica de centros educativos de calidad con orientación inclusiva, se refleja la experiencia desde el quehacer pedagógico de diez instituciones educativas a partir de los fundamentos de educación inclusiva. Enfatizan en la necesidad de que el Ministerio de Educación Pública incorpore el tema de inclusión como componente de la política de calidad.

Las investigaciones mencionadas sugieren el replanteamiento de la praxis educativa direccionada a la consideración de los intereses de la población educativa, de forma que el docente comprenda las necesidades del estudiantado, haciendo uso del principio de equidad educativa, con el cual los docentes faciliten la construcción de espacios de aprendizaje significativos y adecuados al contexto del estudiantado. Así se minimizarán las barreras, las brechas de aprendizaje y motivarán la participación del colectivo estudiantil, a fin de alcanzar el máximo desarrollo cognitivo en el proceso de formación (Arnaiz, 2011).

Enfatizandoenunametodologíadeaprendizaje que brinde oportunidades de aprendizaje para todos, se destacan instituciones que trabajan en disminuir esa brecha digital hacia una educación en atención a la diversidad educativa. Por ejemplo: la Fundación Omar Dengo, con el desarrollo de programas de tecnologías móviles (Castro, 2013), en conjunto con el Ministerio de Educación Pública, ha logrado la apertura de laboratorios de informática educativa, en niveles de preescolar, primaria y secundaria, "fomentando el aprendizaje por proyectos y el desarrollo de habilidades cognitivas, así como el aprendizaje colaborativo" (Castro, 2013, p.133).

Desde esa disminución de la brecha digital, pareciera que el cuerpo docente ha venido trabajando el tema de inclusión muy de la mano con el apoyo de herramientas tecnológicas $\mathrm{y}$, consecuentemente, ha beneficiado a una cantidad de estudiantes. Sin embargo, aún en la actualidad, a pesar de las muchas capacitaciones que se puedan brindar sobre el uso de las TIC para el abordaje educativo de los contenidos temáticos y promover espacios inclusivos de aprendizaje, no se puede negar que hay un porcentaje de docentes que se resisten al cambio. En consecuencia, llevan, desde su quehacer, prácticas educativas inadecuadas que ponen en riesgo el aprendizaje del estudiantado, limitan el conocimiento y potencial que puede desarrollar en clase, por ende, se desfasan de la realidad en la cual se desarrollan (Barrantes y Ross, 2013).

En atención a las razones anteriores y concibiendo el tema de la inclusión en las aulas como un reto existente en la actualidad para el sistema educativo costarricense, la presente investigación adquiere su importancia. Esta surge del interés del estudiantado dela Carrera de Informática Educativa de la Universidad Estatal a Distancia de Costa Rica, comoestudio diagnóstico que da a conocer, con base en la opinión de educadores y estudiantes, si desde los procesos de enseñanza y aprendizaje, en las asignaturas de Ciencias e Inglés, se hace uso pedagógico de recursos tecnológicos disponibles en centros educativos, que promuevan metodologías inclusivas de aprendizaje y favorezcan oportunidades de formación para todos.

Dicho estudio efectúa un análisis comparativo en tres centros educativos, dos públicos y uno privado y desde áreas de enseñanza específicas: ciencias e inglés, para contrastar cómo los docentes de tales áreas desarrollan sus metodologías de aprendizaje, mediante el 
apoyo de los diferentes recursos tecnológicos a disposición, a fin de conocer si los procesos de aprendizaje desarrollados involucran a todo el estudiantado de los grupos a su cargo, para ello, se consideran sus potencialidades, destrezas y se trabaja en función de sus necesidades.

Asípues, lainterrogante sobrelacual se sustenta la investigación y desde la que se expondrán los principales hallazgos es la siguiente: ¿utilizan los docentes de Ciencias e Inglés, de los centros educativos: Llama del Bosque, Juan Flores Umaña y CTP Fernando Volio, herramientas tecnológicas con metodologías de enseñanza inclusivas, que permitan el desarrollo de espacios educativos en atención a la diversidad y que promuevan procesos de formación que involucren las capacidades, necesidades, habilidades y destrezas del estudiantado de sexto y sétimo nivel?

\section{Fundamentación teórica}

El desarrollo de metodologías de enseñanza y aprendizaje que involucren una educación de corte inclusivo, accesible para todos los agentes socioeducativos y en atención a las necesidades e intereses, es una necesidad creciente. Autores, como López y Suarez (Rodríguez, 2017), resaltan características por tomar en cuenta desde los apoyos que recibe el estudiantado en el aula. Señalan que "las potencialidades, ritmos y estilos de aprendizaje son aspectos que todo docente debe considerar en el momento de desarrollar una clase" (p.16).

La incorporación de tales aspectos es posible, desde una mediación que busque la integralidad en el conocimiento; para ello, la gestión del docente desde la metodología de enseñanza y aprendizaje es esencial. Por una parte, Seas (2016) destaca que la metodología de aprendizaje integra varios componentes educativos: técnicas, estrategias, recursos, para la conformación de una dinámica desarrollada desde el interior de una clase. Por otra parte, para Medina y Salvador (2009), una metodología de aprendizaje es aquella acción en la cual se

integran los agentes educativos, una finalidad y conjunto de elementos didácticos, que conllevan a desarrollar una gestión orientada a la puesta en marcha de estrategias, para la consecución de un conocimiento específico gracias a una serie de procesos dados por el docente, en la práctica educativa. (p.173).

En este orden de ideas, es posible apoyar la metodología de aprendizaje con recursos tecnológicos, en aras de facilitar las condiciones de aprendizaje de todo el estudiantado, incluso de aquellos con algún indicador de discapacidad y que deben ser incorporados en el colectivo educativo dentro de un proceso de formación integradora. Para Fernández (Rodríguez, 2017), la señalación hecha es posible, pues las tecnologías se han incorporado en los procesos de aprendizaje como "una oportunidad de mejorar la forma de aprender, involucrando a todo el colectivo educativo y extendiendo los beneficios de ese aprendizaje a todos los territorios y personas" (p. 83).

Para lograrlo, es necesario, además de una mejora en las metodologías de enseñanza y aprendizaje, un cambio de mentalidad en los docentes (Víquez, 2014), de forma que el uso de recursos y de herramientas tecnológicas se incorpore a los procesos de enseñanza y aprendizaje.Asísehacedel procesodeaprendizaje un ecosistema vivencial y que el cuerpo docente involucre una participación más dinamizante, mayor responsabilidad, socialización, pero que, a su vez, le brinde autonomía al estudiantado en su aprendizaje. Estos espacios son idóneos para que el conocimiento sea adquirido, comprendido y aplicado a diferentes realidades (Lugo y Kelly, 2008).

Por su parte, Guerrero (2013) refiere a metodologías, técnicas y recursos que pueden 
utilizarse, desde las aulas, para promover espacios inclusivos de aprendizaje:

- Metodologías y técnicas de aprendizaje cooperativo: tutorías entre iguales, estrategias de colaboración mutua, grupos de apoyo entre docentes, estrategias interactivas de resolución de problemas. Uso de técnicas como: círculos de aprendizaje y TGT (Teams Games Tournaments).

- Metodologías y técnicas de aprendizaje colaborativo: comisiones de apoyo mutuo entre compañeros, aprendizaje desde técnicas como estudios de caso, entrevistas, diálogos, tertulias, discusiones grupales, investigaciones grupales, círculos de acción-reflexión.

- Técnicas de evaluación: pruebas orales, observaciones, diálogos, evaluación de equipo, autoevaluación desde uso de parrillas para la autovaloración de estudiantes, evaluación de evidencias.

- Recursos TIC que pueden apoyar los procesos de aprendizaje: webquest, redes sociales, blogs, foros, portafolios, que favorecen la interacción entre estudiantes y profesor, establecen roles específicos en los estudiantes como liderazgo, colaboración y participación activa. (pp.6-7).

Ahora bien, ante tantos cambios que se exigen desde el campo de acción del cuerpo docente, los cuales involucran una mejora sustancial de su quehacer pedagógico, usando metodologías de enseñanza y aprendizaje con recursos y herramientas tecnológicas que involucren la participación de todo el estudiantado, vale la pena reflexionar sobre los retos que asume el docente del nuevo siglo.

De acuerdo con Borafull, (2014), ser docente en este siglo implica asumir diferentes desafíos, a saber: "significa, aprender a ser guía, motivador, investigador, impulsador, agente creativo $\mathrm{y}$ proveedor de oportunidades de crecimiento para el estudiantado. Se es parte de todo un ecosistema social donde no se puede pretender saberlo todo, más sí estar accesible a asimilar los cambios e innovaciones y participar de ellas con el resto de actores socioeducativos" (p.154).

Se considera fundamental, entonces, que los docentes posean una actitud emprendedora, positiva y flexible para lograr esa amalgama entre el uso de las TIC y el desarrollo de metodologías inclusivas. El hecho de perder el temor ante las innovaciones y, en su lugar, ver las oportunidades de apoyo que los diferentes recursos y herramientas tecnológicas podrían brindar en la mediación pedagógica, conseguiría aprendizajes significativos. Esto se lografía con base en el hecho de ofrecer una educación para todos y adecuando los procesos de mediación a los diferentes estilos de aprendizajes presentes en los estudiantes.

\section{Metodología}

El interés del estudio es profundizar en el contraste de opinión entre el profesorado y el grupo de estudiantes de tres centros educativos costarricenses sobre el uso de las TIC disponibles en cada centro educativo, para determinar si el uso de dichos recursos favorece el desarrollo de metodologías inclusivas de aprendizaje en las asignaturas de Ciencias e inglés. Esto último se descubrirá si se brindan oportunidades de formación para todos y si se consideran las capacidades, las destrezas, los estilos de aprendizaje, las necesidades y las carencias de cada estudiante.

La investigación se destaca por ser descriptiva y explicativa, pues trata de caracterizar una situación dando a conocer las causas de su origen; además, es fenomenológica porque pretende esclarecer el significado que tiene, para los diferentes agentes educativos, el término de inclusión educativa desde un uso de recursos TIC (Bisquerra, 2014). Se sustenta en un enfoque mixto, gracias al diseño exploratorio secuencial con una relación (CUAL-cuan), según los planteamientos de Hernández, Fernández y 
Baptista (2014) y Creswell (2015). Por ser una investigación de tipo mixto, se concentra en una categoría con dos subcategorías y una variable de investigación (ver tabla 1), las cuales serán desarrolladas desde los resultados.

\section{Muestra}

La muestra es no probabilística e intencionada, pues para el estudio, se han considerado los estudiantes con aprobación de sus encargados o padres de familia, mediante el llenado de un consentimiento informado por ser menores de edad. Además, los educadores de las asignaturas de Ciencias e Inglés de los tres centros educativos, para un total de 67 sujetos distribuidos de la siguiente forma: 59 estudiantes en total entre los niveles de sexto y sétimo grado, concentrados en los tres centros educativos, y 8 docentes entre las áreas de conocimiento. Esta distribución se evidencia en las tablas 2 y 3 .

Tabla 1

Categoría y Variable de investigación

\begin{tabular}{lc}
\hline \multicolumn{1}{c}{ Categorías } & Instrumentos \\
\hline Uso de herramientas tecnológicas. & -Entrevistas \\
Metodologías de enseñanza-aprendizaje inclusivas. & -Grupos focales \\
Subcategorías: & -Observaciones \\
-Herramientas tecnológicas usadas en clase. & \\
- Inclusión desde el uso de herramientas tecnológicas. & \\
-Mediación y técnicas usadas en procesos de aprendizaje. & \multicolumn{2}{c}{ Variable } & Instrumento \\
\hline $\begin{array}{l}\text { Comparación de metodologías de enseñanza y aprendizaje en } \\
\text { las asignaturas de Ciencias e Inglés favorecedoras de espacios } \\
\text { inclusivos de aprendizaje. }\end{array}$ & -Cuestionario electrónico \\
\hline
\end{tabular}

Fuente. Ávalos, Redondo, Rojas y Salazar, 2017

Tabla 2

Muestra de investigación estudiantes

\begin{tabular}{|c|c|c|c|c|c|}
\hline \multirow[b]{2}{*}{ Centro educativo } & \multirow[b]{2}{*}{ Rango-edad } & \multicolumn{2}{|c|}{ Género } & \multirow[b]{2}{*}{ Frecuencia } & \multirow[b]{2}{*}{ Porcentaje } \\
\hline & & $\mathrm{H}$ & M & & \\
\hline Llama del Bosque & 11 a 12 años & 5 & 6 & 11 & 18.6 \\
\hline Juan Flores Umaña & 11 a 13 años & 19 & 20 & 39 & 66.1 \\
\hline CTP Fernando Volio & 13 a 14 años & 2 & 4 & 6 & 10.2 \\
\hline *No contestaron & No indica & \multicolumn{2}{|c|}{ No indica } & 3 & 5.1 \\
\hline Total & & 26 & 30 & 59 & 100 \\
\hline
\end{tabular}

Fuente. Cuestionario a estudiantes. 
Tabla 3

Muestra de investigación docentes

\begin{tabular}{lcc}
\hline Centro educativo & Asignatura de Ciencias & Asignatura de Inglés \\
\hline Llama del Bosque & 1 & 1 \\
Juan Flores Umaña & 2 & 2 \\
CTP Fernando Volio & 1 & 1 \\
Total & 4 & 4 \\
\hline
\end{tabular}

Fuente. Entrevista a docentes.

Instrumentos para la recolección de información

Para la recolección de la información, se hicieron entrevistas, procesos de observación mediante escalas Likert y grupos focales. Una vez obtenidos los datos cualitativos, se desarrolló el cuestionario electrónico aplicado a los estudiantes. La validación de los instrumentos se realizó desde el aporte y la revisión por expertos en áreas de tecnología, educación e investigación; se les ofrecieron observaciones a los instrumentos, las cuales fueron consideradas previo a su aplicación.

Tabla 4

Etapas de investigación e instrumentos

\begin{tabular}{lcc}
\hline Etapas & Instrumento aplicado & Actor participante \\
\hline \multirow{3}{*}{ Cualitativa } & Entrevistas & Docentes \\
& Grupos focales & Estudiantes \\
Cuantitativa & Observaciones & Docentes \\
\hline
\end{tabular}

Fuente. Fuente: Ávalos, Redondo, Rojas y Salazar, 2017.

\section{Resultados}

\section{Etapa cualitativa}

Categorías. Uso de las herramientas tecnológicas y metodologías de enseñanza-aprendizaje inclusivas. En esta etapa, se analiza, desde la opinión de los actores educativos en los tres centros educativos, el uso que se les da a las herramientas TIC en las aulas de sexto y sétimo nivel, así como la metodología en el proceso de aprendizaje del estudiantado, empleando los diferentes recursos tecnológicos a disposición.

Los datos obtenidos en las entrevistas a docentes, a grupos focales y a estudiantes son contribuciones privadas y su uso en el tratamiento de la información se elaborará enumerando a los sujetos participantes e indicando el área, no se indican sus nombres.

Para mejorar la comprensión del apartado, se desarrolla la información desde sus dos subcategorías: 


\section{a) Subcategoría 1. Herramientas tecnológicas} usadas en clase

Desde los resultados obtenidos por parte de los docentes en las entrevistas y en los procesos de observación, así como en los aportes de los estudiantes en los grupos focales, se coincide en que tanto las instituciones educativas públicas como la privada sí utilizan recursos tecnológicos en la mediación de las asignaturas de Ciencias e Inglés; sin embargo, desde los aportes de los estudiantes en los grupos focales, se afirma que "se podría interactuar aún más, haciendo más participativas las clases" (estudiantes CTP Fernando Volio, comunicación personal, 25 de abril 2017).

Otros estudiantes afirman, desde los grupos focales: "Todos se motivan cuando ven temas nuevos en clase y se hacen cosas diferentes" (estudiantes de Llama del Bosque, comunicación personal, 25 abril 2017). Lo anterior deja a la vista el aspecto motivacional en el desarrollo de las clases, lo cual pareciera ser un aspecto que condiciona la predisposición de los estudiantes para aprender, y esto se logra mediante las innovaciones que se generen desde el espacio del aula, tal es el caso de procesos de mediación apoyados por variedad de recursos TIC.

Entre los recursos tecnológicos más usados en los tres centros educativos, están los siguientes: celular, conexión de internet institucional, grabadoras, computadoras portátiles con MoviLab, proyectores, YouTube, plataformas educativas virtuales (LMS). Ver tabla 5 de recursos tecnológicos.

A partir de la información de la tabla anterior, se evidencia una mayor disponibilidad de los recursos de hardware por parte del centro educativo privado Llama del Bosque, en comparación con los otros dos centros educativos públicos, donde el docente es quien debe aportar recursos como su computadora personal para disponer de una clase con componentes tecnológicos. Desde los recursos de software, se puede observar una coincidencia de los recursos como YouTube y el uso de aplicaciones de ofimática; sin embargo, se destaca que, a pesar de tener un amplio espacio de información para la búsqueda de actividades y de contar

Tabla 5

Recursos tecnológicos usados en los tres centros educativos

\begin{tabular}{|c|c|c|c|c|}
\hline \multirow[t]{2}{*}{ Centro educativo } & \multirow{2}{*}{$\begin{array}{l}\text { Llama del Bosque } \\
\text { Ciencias e Inglés }\end{array}$} & \multirow{2}{*}{$\begin{array}{c}\text { Juan Flores U } \\
\text { Ciencias e Inglés }\end{array}$} & \multicolumn{2}{|c|}{ CTP Fernando Volio } \\
\hline & & & Ciencias & Inglés \\
\hline Dispositivo hardware & $\begin{array}{l}\text { Calculadora, com- } \\
\text { putadora, proyector, } \\
\text { tabletas y celulares. }\end{array}$ & $\begin{array}{l}\text { Celular, proyector, } \\
\text { computadora del } \\
\text { personal docente. }\end{array}$ & $\begin{array}{l}\text { Celular, proyector, } \\
\text { computadora per- } \\
\text { sonal del docente. }\end{array}$ & $\begin{array}{l}\text { Computadoras } \\
\text { CD con activida- } \\
\text { des, proyector, } \\
\text { celular. }\end{array}$ \\
\hline Dispositivo software & $\begin{array}{l}\text { YouTube, educare- } \\
\text { digital. com, plata- } \\
\text { forma Santillana, } \\
\text { Word, Power Point. }\end{array}$ & $\begin{array}{l}\text { Códigos QR; You- } \\
\text { Tube, actividades } \\
\text { digitales del libro de } \\
\text { texto. }\end{array}$ & $\begin{array}{l}\text { YouTube, busca- } \\
\text { dores, bibliotecas } \\
\text { digitales. }\end{array}$ & $\begin{array}{l}\text { Apps, Word } \\
\text { references, You- } \\
\text { Tube, actividades } \\
\text { digitales, Power } \\
\text { Point, Word. }\end{array}$ \\
\hline
\end{tabular}

Fuente. Ávalos, Redondo, Rojas y Salazar, 2017 
con el acceso a internet, se limitan mucho al uso de bibliotecas digitales o a bases de datos de información cuando pudieran usar otros espacios para generar espacios de mediación más dinámicos e inclusivos, por ejemplo, herramientas de gamificación o de ludificación: Brainscape, Cerebriti, Minecraft: Education Edicion y ChemCaper, entre muchas otras existentes, las cuales dinamizan el aprendizaje de los estudiantes mediante la puesta en marcha de los conocimientos teóricos adquiridos, incluso aprenden gracias a la práctica del hacer desde el desarrollo de juegos educativos vinculados al contenido teórico visto en la clase.

Otros aspectos por resaltar en las entrevistas hechas a los profesores de los diferentes centros educativos vinculados con las TIC es sobre su anuencia e interés de ser capacitados. En este sentido, de los ocho docentes entrevistados, la mayoría están muy anuentes a capacitarse de forma contínua para mejorar su quehacer en el aula, pues son conscientes del poco conocimiento que poseen en el uso de herramientas didácticas digitales. Solo uno de los docentes no mostró ese interés por capacitarse, o bien usar estos recursos como apoyo de las clases, pues indicó que "abarcarían su tiempo de trabajo y no avanzaría en el desarrollo de contenidos" (docente 2 de Inglés, centro educativo Juan Flores Umaña, comunicación personal, 14 de abril, 2017).

b) Subcategoría 2. Inclusión desde el uso de herramientas tecnológicas

Un último aspecto por resaltar es sobre el uso inclusivo de las herramientas tecnológicas y cómo lo logran, aspectos que fueron consultados a los docentes en las entrevistas. Los entrevistados manifestaron variedad de opiniones:

En el caso del centro educativo Llama del Bosque, seafirmaque“...cadaestudiantetieneuna participación activa en las actividades realizadas con actividades tecnológicas..." (docentes
Llama del Bosque, comunicación personal, 14 de abril, 2017). Estos aportes son consecuentes con las observaciones y grupos focales.

Desde la opinión de los docentes del centro educativo Juan Flores Umaña, las docentes de Inglés indicaron, por un lado: "Se usan herramientas que estimulan distintas destrezas para satisfacer las diversas necesidades y estilos de aprendizaje, como videos, canciones, aplicaciones de los alumnos, entre otros" (docente 1 de Inglés, 14 de abril, 2017). Por otro lado, la docente 2 hace un uso inclusivo cuando "utilizo el video beam con temas relacionados al programa de estudio del MEP y de acuerdo al nivel" (docente 2 de Inglés, comunicación personal, 14 de abril, 2017).

En el caso de los docentes de ciencias de la misma institución (Juan Flores Umaña), indicaron usar, de forma inclusiva, los recursos tecnológicos:

...como medio de motivación o ampliación de temas a ver en las asignaturas, además se le permite al estudiantado usar el teléfono en ejercicios que se pueda aprovechar. Se ingresa a internet y otros como calculadora para la resolución de problemas, ampliar vocabulario ... (docente 1 de Ciencias, comunicación personal, 14 de abril, 2017).

El uso de esos recursos sí fue constatado desde las observaciones, en las cuales se vio el uso del celular con propósitos educativos, en un espacio de aprendizaje motivado hacia la participación activa y el diálogo.

Finalmente, la docente 2 de ciencias del centro educativo Juan Flores Umaña indicó el uso inclusivo "a la hora de proyectar un video relacionado con el tema" (docente 2 de Ciencias, comunicación personal, 14 de abril, 2017).

A pesar de esas opiniones sobre el empleo positivo de las tecnologías, se resalta que, desde lo observado en las clases respecto al uso de los 
recursos tecnológicos y aportes de los estudiantes en los grupos focales, la mediación resulta ser diferente a la reportada por los docentes del centro educativo Juan Flores Umaña en las entrevistas, pues no todos los docentes utilizan los recursos que mencionan. Como se ha dicho, esto se evidenció desde las observaciones, pues el libro de texto y el desarrollo de actividades dirigidas en el aula son los recursos de uso más frecuente para el abordaje de los contenidos.

En el caso de los docentes del CTP Fernando Volio, por una parte, la docente de inglés indica que sí realiza la inclusión en el uso de los recursos "cuando se trata de ayudar al estudiante de acuerdo con sus necesidades especiales evaluando dudas individuales y brindándoles asesoría" (docente de Inglés, comunicación personal, 14 de abril, 2017). Por otra parte, la de ciencias afirma brindar el trabajo inclusivo de las herramientas y ofrece ejemplos claros de ese uso: "El apoyo del celular de los estudiantes para hacer investigaciones durante las lecciones. El uso de la computadora para reproducir videos que fortalezcan el aprendizaje" (docente de Ciencias, comunicación personal, 14 de abril, 2017). Aspecto que se evidencia claramente desde los procesos de observación, pues promueven una participación activa e investigativa por parte del estudiantado en las clases.

c) Subcategoría 3. Mediación y técnicas usadas en procesos de aprendizaje

En esta subcategoría, se enfatizó en la mediación desarrollada por los docentes de los diferentes centros educativos en las asignaturas de Ciencias e Inglés, además del uso de técnicas y de estrategias que sustentan toda una metodología de aprendizaje orientada a la enseñanza desde la inclusión. Para ello, se reunieron los resultados de las entrevistas a docentes y los de los cuestionarios aplicados a los estudiantes (información relacionada con la tabla 6), así como los aportes de grupos focales. Las respuestas se concentraron en relación con los siguientes aspectos:

A partir de los indicadores establecidos, se constató, de acuerdo con la opinión de los docentes, que, en la metodología y técnicas empleadas en clase, ellos destacan por ser actores educativos activos, agentes motivadores y sugerentes de una participación activa por parte del estudiantado, gracias a variadas

\section{Tabla 6}

Indicadores sobre mediación y técnicas usadas por los docentes

Aspectos por valorar en el cumplimiento de la inclusión

Metodología y técnicas usadas en clase

Seguimiento y evaluación de los aprendizajes
Indicadores

-Sobre las clases desarrolladas por el docente

-Sobre el uso de trabajo colaborativo

-Uso de técnicas de investigación

-Ambiente de clase

-Seguimiento y evaluación

Conocimientos previos

-Consideración de habilidades y destrezas

-Seguimiento de la evaluación

-Realimentación que reciben

Fuente. Datos del cuestionario electrónico. 
actividades que integran al colectivo estudiantil y que, además, tal metodología usada se apoya de diferentes herramientas tecnológicas.

Solo una docente de Ciencias de nivel de sexto grado de la institución Juan Flores Umaña no contesta favorablemente sobre el desarrollo de la metodología empleada en sus clases. El resto de docentes (siete) afirma emplear técnicas para promover el trabajo colaborativo como estrategia que integra al estudiantado.

Estas últimas opiniones fueron confrontadas con los resultados del cuestionario aplicado a estudiantes (en tabla 6), en el cual más del $70 \%$ coincide con los planteamientos anteriores brindados por el personal docente en las entrevistas. El estudiantado afirma que los docentes sí son actores que los motivan a participar, sugieren actividades variadas para desarrollar en las clases e integran al grupo en el desarrollo de tales actividades, las cuales tienen apoyo de recursos tecnológicos.

Situados en el mismo indicador sobre metodología y técnicas usadas en clase, se les consultó acerca del empleo de técnicas de investigación, como la observación o el aprendizaje contextualizado. Únicamente, un educador de Ciencias de nivel de sexto grado de la institución Llama del Bosque indicó "no usar dichas técnicas investigativas, en razón de que es un grado escolar y la mayoría del material didáctico es facilitado al estudiantado; sin embargo, señaló que sí se intenta desarrollar la capacidad de análisis" (docente de Ciencias de Llama del Bosque, comunicación personal, 14 de abril, 2017).

El resto de los docentes de Ciencias e Inglés de los otros dos centros educativos afirman sí usar técnicas investigativas, como la observación y el trabajo contextualizado en sus clases. No obstante, desde la opinión de los estudiantes, la información varía, pues del total que completaron el cuestionario en las tres instituciones, $66 \%$ afirma la presencia de este tipo de técnicas en las clases, mientras que hay un dato de interés reflejado entre los que se encontraron dudosos $(17 \%)$, quienes no coincidieron con el uso de estas técnicas en clase $(12 \%)$ y aquellos que no contestaron la pregunta (5\%). Esto hace pensar en una opinión muy dividida entre el uso de técnicas de investigación y quienes en realidad no perciben el uso de dichas técnicas en su aprendizaje.

Desde el siguiente indicador, sobre seguimiento y evaluación de los aprendizajes, uno de los docentes de nivel de sétimo año del centro educativo CTP Fernando Volio, de la asignatura de Ciencias, no tomó en cuenta los conocimientos previos de los estudiantes para reforzar los nuevos conocimientos, lo cual apunta a una contradicción del mismo docente, pues en otro punto del mismo indicador referido a si se consideran las opiniones de los estudiantes para el desarrollo de las actividades en clase (ver tabla 6), el mismo docente había afirmado que sí tomaba en cuenta las ideas, las valoraciones y las sugerencias para el desarrollo de las actividades.

Siguiendo con el análisis del mismo indicador sobre la evaluación de los aprendizajes, otro docente, pero esta vez de la asignatura de Inglés de nivel de sexto y del centro educativo Juan Flores Umaña, evidencia, en comparación con el resto de los docentes entrevistados, que no realiza desde el inicio del período lectivo pruebas diagnósticas para conocer aspectos sobre el aprendizaje de los estudiantes, fortalezas y debilidades, y así, dar paso a un planeamiento curricular educativo inclusivo.

Lo planteado hace pensar que, quizás, para las clases y actividades sí se brinda el espacio de opinión a los estudiantes, pero no se dan los espacios completos de seguimiento en la evaluación educativa. ¿Cuáles son las razones? Se hacen inferencias por parte de las 
investigadoras sobre factores como el tiempo y la carga académica; sin embargo, el proceso de evaluación desarrollado adecuadamente es fundamental para conocer cuánto saben los estudiantes de determinado tema, y no está siendo desarrollado. Esto podría afectar en cómo podría ser abordado el conocimiento desde las diferentes actividades en clase, pues no es están considerando los diferentes estilos de aprendizaje con los que cuentan los estudiantes.

Adicionalmente, en relación con las opiniones de los docentes, pareciera haber un desconocimiento o confusión de la importancia que tiene el proceso de evaluación desde sus funciones (Pérez, 2006), ya que por un lado, no se concibe conocer los aprendizajes previos sin aplicar el proceso de evaluación diagnóstica. Por otro lado, es imposible desconocer, desde la práctica educativa, cuál es la forma de aprendizaje predominante de cada estudiante. Es deber de todo docente conocer a fondo las características socioeducativas de los estudiantes para propiciar un adecuado proceso de aprendizaje (Medina, 2009), sobre todo, si se espera que sea de carácter inclusivo.

Otro aspecto por resaltar, desde la evaluación de los aprendizajes, es la consideración de las habilidades y destrezas de los estudiantes para la mejora del proceso de aprendizaje en las clases. Ante esto, todos los docentes entrevistados, tanto de sexto como de sétimo y en las dos asignaturas, afirmaron tomar en cuenta tales habilidades y destrezas del estudiantado; sin embargo, tal aseveración, una vez más, sugiere ser una contraposición de opiniones con los resultados de los estudiantes, pues desde las respuestas encontradas en el cuestionario, 56\% de los alumnos parece no coincidir con las aportaciones de los docentes.

Se les consultó si se da un seguimiento al aprendizaje del estudiante, en el cual se les señale qué conocimientos deben mejorar y cómo lograrlo. Desde las entrevistas, la mayoría de los docentes entrevistados afirmaron desarrollar este proceso en sus clases; no obstante, el docente de Ciencias de sétimo nivel del colegio CTP Fernando Volio condicionó la respuesta indicando "depende del objetivo y según sea este, entonces, da esa mediación al estudiante..." (docente de Ciencias, comunicación personal,14 abril, 2017).

Lo respuesta anterior nos hace pensar el porqué de esa condición: cuando es muy necesaria la mediación del docente hacia el estudiante, sobre todo, en la forma en cómo pueda mejorar las prácticas erradas y generar nuevas (Martínez, 2007), debido a que tales espacios permiten reforzar y mejorar habilidades y destrezas en determinado conocimiento.

Dentro de ese mismo aspecto, las respuestas de los estudiantes favorecen y coinciden con los docentes en $70 \%$, pues afirman que sí se ejecuta esta acción en las asignaturas de Ciencias e Inglés. Lo mismo sucede con el aspecto de realimentación, tanto los estudiantes como los docentes coinciden en que sí se ofrece una buena realimentación para mejorar o ampliar el aprendizaje de los estudiantes.

Desde el uso de la evaluación desde sus tipos: autoevaluación, coevaluación y evaluación unidireccional en los diferentes momentos de aprendizaje, cinco de ocho docentes afirman aplicar estos procesos en sus clases. Los docentes del centro educativo Llama del Bosque, así como del CTP Fernando Volio, en la asignatura de Inglés, condicionaron su respuesta a no usar del todo dichos tipos de evaluación. Desde la opinión de los estudiantes, los resultados alarman, pues más de la mitad de la muestra participante afirma no estar de acuerdo con lo dicho por los docentes; es decir, desde la perspectiva de los estudiantes, más de la mitad no consideran que los docentes empleen las evaluaciones en los diferentes momentos de aprendizaje. 


\section{Etapa cuantitativa}

Variable: Comparación de metodologías de enseñanza y aprendizaje en las asignaturas de Ciencias e Inglés, favorecedoras de espacios inclusivos de aprendizaje. Para su abordaje, se enfatizó en la mediación desarrollada por los docentes, desde los diferentes centros educativos en estudio. Se consideraron los resultados de las entrevistas a docentes, a partir del apartado de metodología y técnicas usadas en clase y del seguimiento y evaluación de los aprendizajes en contraste con los resultados del cuestionario aplicado a los estudiantes. Esta información se detalla en la tabla 7.

Tabla 7

Comparación de metodologías inclusivas en las asignaturas de Ciencias e inglés

\begin{tabular}{ccccc}
\hline Indicadores/ Centro educativo & $\begin{array}{c}\text { Llama del } \\
\text { Bosque }\end{array}$ & $\begin{array}{c}\text { Juan Flores } \\
\text { Umaña }\end{array}$ & $\begin{array}{c}\text { CTP } \\
\text { Fernando } \\
\text { Volio }\end{array}$ & $\begin{array}{c}\text { Opinión } \\
\text { estudiantes }\end{array}$ \\
\hline
\end{tabular}

\begin{tabular}{rcccccccccr}
\hline Metodología y técnicas usadas en la clase & I 7 & C6 & I6 & C6 & C6 & I6 & I7 & C7 & Resultados \\
\hline El(la) educador (a) motiva a los estudiantes, & $\sqrt{ }$ & $\sqrt{ }$ & $\sqrt{ }$ & $\sqrt{ }$ & $\sqrt{ }$ & $\sqrt{ }$ & $\sqrt{ }$ & $\sqrt{ }$ & Más del 70\% Sí.
\end{tabular}
sugiere espacios de participación integradoras y apoya las clases con el uso de las TIC.

Promueve el trabajo de grupo como técnica para integrar a todos sus estudiantes.

Hace uso de técnicas como: investigación, observación, aprendizaje contextualizado, apoya de recursos tecnológicos para un aprendizaje a largo plazo.

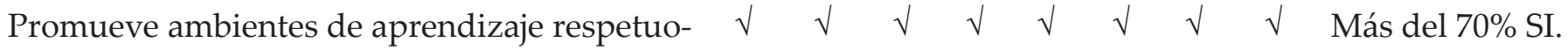
sos y colaboradores, incentivando a diario la participación del estudiantado.

¿Toma en cuenta las ideas, valoraciones y sugerencias del estudiantado para el desarrollo de las actividades en la clase?

$\begin{array}{lllllllll}\sqrt{ } & \sqrt{ } & \sqrt{ } & \sqrt{ } & \mathrm{NC} & \sqrt{ } & \sqrt{ } & \sqrt{ } & \begin{array}{l}68 \% \mathrm{SI}, 20 \% \mathrm{Du}- \\ \text { dan, } 9 \% \mathrm{NO}, 3 \% \\ \mathrm{NC}\end{array} \\ & & & & & & & \\ \sqrt{ } & \mathrm{NO} & \sqrt{ } & \sqrt{ } & \sqrt{ } & \sqrt{ } & \sqrt{ } & \sqrt{ } & \begin{array}{l}66 \% \mathrm{SI}, 17 \% \text { dudan } \\ 12 \% \mathrm{No}, 5 \% \mathrm{NC} .\end{array}\end{array}$

$\begin{array}{llllllllll} & \sqrt{ } & \sqrt{ } & \sqrt{ } & \sqrt{ } & \sqrt{ } & \sqrt{ } & \sqrt{ } & \sqrt{ } & 64 \% \text { SI, } 17 \% \text { dudan. }\end{array}$ $15 \% \mathrm{NO}, 4 \% \mathrm{NC}$.

\section{Seguimiento y evaluación de los aprendizajes}

Toma en cuenta los conocimientos previos de los estudiantes, y refuerza nuevos.

Realiza pruebas de diagnóstico para conocer cuál es la mejor forma de aprender del estudiantado: (visual, auditiva, escrita, oral.).

\begin{tabular}{|c|c|c|c|c|c|c|c|c|}
\hline$\sqrt{ }$ & $\sqrt{ }$ & $\sqrt{ }$ & $\sqrt{ }$ & $\sqrt{ }$ & $\sqrt{ }$ & $\sqrt{ }$ & $\mathrm{NO}$ & $\begin{array}{l}68 \% \text { SI, } 17 \% \text { du- } \\
\text { dan, } 10 \% \text { NO, } 5 \% \\
\text { NC. }\end{array}$ \\
\hline$\sqrt{ }$ & $\sqrt{ }$ & $\sqrt{ }$ & $\sqrt{ }$ & $\sqrt{ }$ & NO & $\sqrt{ }$ & $\sqrt{ }$ & $\begin{array}{l}42 \% \text { Si, 39\% NO, } \\
14 \% \text { dudan, } 5 \% \\
\text { NC. }\end{array}$ \\
\hline
\end{tabular}

Continúa... 


\begin{tabular}{|c|c|c|c|c|c|c|c|c|c|}
\hline $\begin{array}{l}\text { Considera las habilidades y destrezas del es- } \\
\text { tudiantado para mejorar el proceso de apren- } \\
\text { dizaje en las clases. }\end{array}$ & $\sqrt{ }$ & $\sqrt{ }$ & $\sqrt{ }$ & $\sqrt{ }$ & $\sqrt{ }$ & $\sqrt{ }$ & $\sqrt{ }$ & $\sqrt{ }$ & $\begin{array}{l}44 \% \text { SI, } 37 \% \text { du- } \\
\text { dan, } 14 \% \text { NO, } 5 \% \\
\text { NC. }\end{array}$ \\
\hline $\begin{array}{l}\text { Da un seguimiento al aprendizaje del educan- } \\
\text { do, les hace saber qué conocimientos deben } \\
\text { mejorar y cómo realizar esas mejoras. }\end{array}$ & $\sqrt{ }$ & $\sqrt{ }$ & $\sqrt{ }$ & $\sqrt{ }$ & $\sqrt{ }$ & $\sqrt{ }$ & $\sqrt{ }$ & $\sqrt{ }$ & Más del 70\% SI. \\
\hline $\begin{array}{l}\text { La realimentación ofrecida, contribuye a me- } \\
\text { jorar, ampliar o reforzar el aprendizaje de los } \\
\text { estudiantes en los temas de la asignatura que } \\
\text { imparte. }\end{array}$ & $\sqrt{ }$ & $\sqrt{ }$ & $\sqrt{ }$ & $\sqrt{ }$ & $\sqrt{ }$ & $\sqrt{ }$ & $\sqrt{ }$ & $\sqrt{ }$ & Más del 70\% SI. \\
\hline $\begin{array}{l}\text { ¿Se establecen desde el inicio de cada activi- } \\
\text { dad, indicaciones claras para su desarrollo y se } \\
\text { le informa al estudiante cómo será evaluado? }\end{array}$ & $\sqrt{ }$ & $\sqrt{ }$ & $\sqrt{ }$ & $\sqrt{ }$ & $\sqrt{ }$ & $\sqrt{ }$ & $\sqrt{ }$ & $\sqrt{ }$ & $\begin{array}{l}52 \% \text { SI, 37\% dudan, } \\
8 \% \text { No, 3\% NC. }\end{array}$ \\
\hline $\begin{array}{l}\text { En sus clases hace uso de la autoevaluación, } \\
\text { coevaluación y evaluación unidireccional, des- } \\
\text { de los diferentes momentos de aprendizaje. }\end{array}$ & $\sqrt{ }$ & $\mathrm{NO}$ & $\sqrt{ }$ & $\sqrt{ }$ & $\sqrt{ }$ & $\sqrt{ }$ & $\sqrt{ }$ & $\sqrt{ }$ & $\begin{array}{l}44 \% \text { SI, } 25,4 \% \\
\text { dudan, } 25,4 \% \text { No, } \\
5 \% \text { NC. }\end{array}$ \\
\hline
\end{tabular}

Nota. I6: Inglés sexto; I7: Inglés sétimo; C6: Ciencias sexto; C7: Ciencias sétimo; NC: No contesta, ( $\sqrt{ })$ : si se realiza. Fuente: Datos cuestionario electrónico.

Los datos anteriores permiten resaltar los siguientes aportes:

Desde el indicador sobre uso de técnicas de investigación en el aula: en opinión de los docentes, más de $90 \%$ indica que sí usa las técnicas señaladas, como observación, aprendizaje contextualizado; sin embargo desde los estudiantes, 17\% duda de esa aplicación en las aulas, $12 \%$ refiere que tales técnicas no se usan y $5 \%$ no responde.

Los resultados del indicador sobre consideración de ideas, valoraciones y sugerencias del estudiantado, en el desarrollo de las actividades de clase, evidencian que, según aportes de los docentes, $100 \%$ indica que sí toma en cuenta las ideas de los estudiantes. Este resultado difiere de los aportes de los alumnos, pues aunque $64 \%$ indica que sí se realiza ese indicador, $17 \%$ duda de ese aspecto, $15 \%$ niega su realización y $4 \%$ no aporta al instrumento.
En el caso del indicador que refiere a la ejecución de las pruebas de diagnóstico para conocer cuál es la mejor forma de aprender del estudiantado, $90 \%$ de los docentes señalan que sí ejecutan las pruebas, pero los estudiantes presentan diferentes opiniones: 39\% señala que no se aplican esas pruebas, $14 \%$ duda de ese aspecto y $5 \%$ no respondió a tal indicador.

En el caso del último indicador, sobre los tipos de evaluación que se aplican en los procesos de aprendizaje, $90 \%$ de los docentes indican que sí hacen uso de todos los tipos de evaluación, pero nuevamente el estudiantado difiere: más de $50 \%$ no está de acuerdo con ese indicador. Por consiguiente, las respuestas de los educadores no coinciden con las de los entrevistados.

Los resultados anteriores sugieren se haga una reflexión sobre el papel del estudiante en los procesos de aprendizaje y, desde la gestión, el de los educadores si realmente los espacios de mediación que los docentes consideran inclusivos son percibidos de igual forma por 
los estudiantes. Esto porque las opiniones de los estudiantes, en la mayoría de los casos, no coincidieron con los aportes de los docentes; por lo tanto, pareciera ser necesario buscar un punto de acuerdo entre los agentes educativos y que se cambie la forma de ver la educación.

Este no debe ser un aspecto jerárquico donde el educador es quien tiene la última palabra, mas sí que sea un espacio que brinde oportunidades de aprendizaje para todos entendiendo que el aprendizaje es un derecho para todos e involucra a todos en igualdad de condiciones (Arnaiz, 2003). El centro educativo debe adecuarse a las necesidades e intereses del estudiante y no al revés.

\section{Conclusiones}

Uno de los principales hallazgos de la investigación, en el contraste de opiniones entre estudiantes y educadores, ha sido la ausencia de la práctica inclusiva en el aprendizaje que reciben los estudiantes. Estos últimos revelaron que los docentes no hacen uso inclusivo ni pedagógico de los recursos tecnológicos, sino más bien un uso instrumental, por ejemplo: al transmitir información desde la proyección de un video o presentación, no demuestran, en la mediación, metodologías ni técnicas inclusivas de acuerdo con planeamientos señalados por Guerrero (2013).

Desde la comparación de metodologías de enseñanza, es importante que se direccionen los procesos de enseñanza-aprendizaje a espacios menos jerárquicos y de mayor enfoque colaborativo, pues es justamente en la socialización de ideas y en el trabajo colectivo que se esclarecen los problemas de aprendizaje, se atienden las debilidades y se fomenta el interés de cada estudiante, para generar aprendizajes cognitivos y para la vida (Schank, 2011) (El texto original es en inglés).
A partir de los resultados obtenidos y del contraste de la teoría, pareciera que la falta de iniciativa, el planeamiento inflexible, el desconocimiento en el tema de inclusión desde el empleo de prácticas de aprendizaje apoyadas por diferentes recursos tecnológicos y la resistencia al cambio para implementar nuevas metodologías de aprendizaje podrían figurar como posibles causas que imposibilitan el desarrollo de una gestión de aprendizaje de corte inclusivo desde la mediación realizada por los docentes en las asignaturas de Ciencias e Inglés.

Se coincide en la importancia de sensibilizar a las poblaciones educativas en el tema de inclusión y de atención a la diversidad, para que se entienda que dicha acción debe estar presente en las aulas y formar parte de todos los elementos educativos que integran el proceso de enseñanza y aprendizaje, pues todos somos parte de una diversidad con particularidades, fortalezas y debilidades, lo que enriquece el espacio de aula en la formación de valores y de nuevos saberes desde una integralidad propiciada por la encomiable gestión de los educadores.

En razón de lo anterior, es esencial que los docentes se capaciten para implementar, en el aula, metodologías y técnicas de aprendizaje verdaderamente inclusivas que, apoyadas en el uso efectivo de la tecnología, permitan entender y trabajar la dinámica de aprendizaje en función de las necesidades de los estudiantes. Ante ello, se sugiere valorar los aportes y los beneficios que ofreceel método DiseñoUniversaldeAprendizaje (DUA), el cual podría favorecer, desde una reestructuración curricular en la metodología, los contenidos, las técnicas, los materiales y el uso de recursos tecnológicos hasta el desarrollo de procesos de aprendizaje, en los cuales el proceso educativo esté al alcance y a la disposición de todos los agentes educativos (Pastor, 2012, en Navarro, Fernández, Soto y Tortosa, 2012). 


\section{Referencias}

Ainscow, M.; Booth, T. \& Dyson, A. (2006). Improving schools, developing inclusion. Londres: Routledge.

Arnaiz, P. (2003) Educación inclusiva: una escuela para todos. España: Aljibe.

Arnaiz, P. (2011). Escuelas eficaces e inclusivas: cómo favorecer su desarrollo. Educatio Siglo XXI, 1(30), 25-44.

Ávalos, A. (agosto, 2015). En otros países ofrecen más oportunidades a los superdotados. (Publicación en prensa). Recuperado de https://www.nacion.com/el-pais/educacion/ en-otros-paises-se-ofrecen-mas-oportunidades-a-los-superdotados/FIIRY7WQLJDVJOKZLJL34QA6HA/story/

Barrantes, A. y Ross, A (febrero,2013). Tecnologías llegan al aula con débil capacitación de docentes. (Noticia en prensa). Recuperado de http://www.nacion.com/el-pais/servicios/ tecnologias-llegan-al-aula-con-debil-capacitacion-de-docentes/GSEB72PRNFBQ7HFXXRFACWU5FI/story/

Bisquerra, R. (2014) Metodología de la Investigación Educativa. Cuarta edición. Madrid: LA MURALLA S.A.

Borafull, I. (2014). El futuro de la educación vinculado a un nuevo modelo productivo en una sociedad de cambios disruptivos. Revista de Humanidades, 13(2),150-165. Recuperado de https://www.fundacionpfizer.org/sites/default/files/pdf/dendra_ nov_2014_01_El_futuro_de_la_educacion. pdf

Castro, H. (2013). Proceso de gestión del Proyecto de Tecnologías Móviles: Estudio de caso. Revista Gestión de la Educación, 1(4),103-137.

Castro, J. (2013). La implementación de prácticas inclusivas en instituciones educativas del Mi- nisterio de Educación Pública de la Región de Heredia. (Trabajo Final de Graduación para optar por el grado de Máster en Psicopedagogía). Universidad Estatal a Distancia. San José Costa Rica.

Creswell, J. (2015). A Concise Introduction to Mixed Methods Research. Los Angeles. United States of America. SAGE.

Duryea, S. y Robles, M. (2016) Realidades y Perspectivas. Pulso Social de América Latina y el Caribe 2016. Informe del Banco Interamericano de Desarrollo (BID). Recuperado de https://publications.iadb.org/ handle/11319/7863?locale-attribute=es\&locale-attribute $=\mathrm{en}$

Guerrero, C. (julio, 2012). Hacia la construcción de procesos y prácticas "exclusivas": Metodologías para la intervención. Quaderns d'Animació i Educació Social, 16, ISSN: 1698-4404. Recuperado de http://quadernsanimacio.net/ANTERIORES/diciseis/arti2_ practicas\%20exclusivas.pdf

Hernández, R., Fernández, C. y Baptista, P. (2014) Metodología de la Investigación. Sexta Edición. México: Mc Graw Hill.

Lugo, M. y Kelly, V (2008) La gestión de las TIC en las escuelas: el desafío de gestionar la innovación. Ponencia. Seminario internacional: Cómo las TIC transforman las escuelas. UNICEF Argentina. Recuperado de https://www.unicef.org/argentina/spanish/IIPE_Tic_06.pdf

Marín, M. y Meléndez, L. (2011). Proyecto: construyamos centros educativos inclusivos. Un modelo para evaluar actitudes, políticas y acciones institucionales. Innovaciones educativas, 18(13), 1-10. Recuperado de http://investiga.uned.ac.cr/revistas/index. php/innovaciones/article/view/577

MEP (Ministerio de Educación Pública). (2008). El Centro Educativo de Calidad como 
eje de la Educación Costarricense. Universal. San José, Costa Rica. Recuperado de http:// www.uned.ac.cr/ece/images/documents/ doc2011_yrivera/un_centro_educativo_de_ calidad.pdf

Martínez, C. (2007). Evaluación de programas. Modelos y procedimientos. Madrid: UNED.

Meléndez, L. (2015) Situación actual de los Centros de Educación Especial. Informe Final del Estado de la Nación Costa Rica. Recuperado de https://estadonacion.or.cr/files/biblioteca_virtual/educacion/005/Lady_Melendez_ et_al_Situacion_actual_centros_de_educ_ espec.pdf

Medina, A. (2009) Didáctica. Formación básica para profesionales de la educación. Madrid España: UNIVERSITAS.

Medina, A. y Salvador, F. (2009). Didáctica General. Madrid: Pearson Education.

Navarro, J.; Fernández, M.T.; Soto, F. J. \& Tortosa, F.(coords). (2012). Respuestas flexibles en contextos educativos diversos. Murcia: Consejería de Educación, Formación y Empleo.

Pérez, R. (2006). Evaluación de programas educativos. Madrid: La Muralla.

Rodríguez, A. (comp.) (2017). Prácticas Innovadoras inclusivas: retos y oportunidades. Oviedo: Universidad de Oviedo.
Seas, J. (2016). Didáctica General 1. San José, Costa Rica. EUNED.

Segura, M. y Martínez, R. (2014) Proyecto: Apoyo a la gestión pedagógica a centros educativos de calidad con orientación inclusiva. Conexiones, una experiencia más allá del aula. Ministerio de Educación Pública, 3(6), 15-23. Recuperado de: http://www. mep.go.cr/sites/default/files/3revistaconexiones2014.pdf

Schank, R. (2011) Teaching Minds: How Cognitive Science Can Save Our Schools. New York Teachers College Press.

Organización de las Naciones Unidas para la Educación, la Ciencia y la Cultura (UNESCO) (1994). Declaración de Salamanca y Marco de acción sobre Necesidades Educativas Especiales. Acceso y Calidad. Ministerio de Educación y Ciencia España: UNESCO. Recuperado de: www.unesco.org/education/pdf/ SALAMA_S.PDF

Víquez, I. (noviembre, 2014). Las TIC en la educación inclusiva e influencia en el aprendizaje de preescolares. Congreso Iberoamericano de Ciencia, Tecnología, Innovación y Educación. Buenos Aires Argentina. Recuperado de http://www.oei.es/historico/congreso2014/memoriactei/675.pdf

Recibido: 14 de febrero de 2018 Aceptado: 26 de abril de 2018 
